\title{
A Gallium Oxide-Graphene Oxide Hybrid Composite for Enhanced Photocatalytic Reaction
}

\author{
Seungdu Kim ${ }^{1,2}$, Kook In Han ${ }^{1}$, In Gyu Lee ${ }^{1}$, Won Kyu Park ${ }^{2,3}$, Yeojoon Yoon ${ }^{2}$, Chan Sei Yoo ${ }^{2}$, \\ Woo Seok Yang ${ }^{2, *}$ and Wan Sik Hwang ${ }^{1, *}$ \\ 1 Department of Materials Engineering, Korea Aerospace University, Goyang 412-791, Korea; \\ seungdukim@gmail.com (S.K.); kooookin@gmail.com (K.I.H.); leeig@kau.ac.kr (I.G.L.) \\ 2 Electronic Materials and Device Research Center, Korea Electronics Technology Institute, Seongnam 463-816, \\ Korea; pwkstyle@gmail.com (W.K.P.); yajoony@keti.re.kr (Y.Y.); ychs@keti.re.kr (C.S.Y.) \\ 3 School of Advanced Materials Science and Engineering, Sungkyunkwan University, Suwon 440-746, Korea \\ * Correspondence: wsyang@keti.re.kr (W.S.Y.); whwang@kau.ac.kr (W.S.H.); \\ Tel.: +82-31-789-7256 (W.S.Y.); +82-2-300-0293 (W.S.H.)
}

Academic Editors: Ming-Tsang Lee and Te-Hua Fang

Received: 1 June 2016; Accepted: 23 June 2016; Published: 1 July 2016

\begin{abstract}
Hybrid composites (HCs) made up of gallium oxide (GaO) and graphene oxide (GO) were investigated with the intent of enhancing a photocatalytic reaction under ultraviolet (UV) radiation. The material properties of both $\mathrm{GaO}$ and $\mathrm{GO}$ were preserved, even after the formation of the HCs. The incorporation of the GO into the GaO significantly enhanced the photocatalytic reaction, as indicated by the amount of methylene blue (MB) degradation. The improvements in the reaction were discussed in terms of increased surface area and the retarded recombination of generated charged carriers.
\end{abstract}

Keywords: gallium oxide; graphene oxide; photocatalytic reaction; methylene blue; ultraviolet radiation

\section{Introduction}

Various metal oxides have been investigated with respect to their photocatalytic reactions, and the resulting technologies can already be found in self-cleaning and anti-fogging/antibacterial fields $[1,2]$. These products work via the photo-induced redox reactions of adsorbed materials and/or photo-induced hydrophilic conversion of materials [3-5]. The acceleration of the photoreaction occurs on a material's surface, so the introduction of nano-materials and nano-technologies can significantly enhance catalytic efficiency [6-9]. Meanwhile, two-dimensional (2D) materials, such as graphene and graphene oxide, have been intensively studied in various areas due to their extraordinary high surface-area to volume ratio [10]. Theoretically, a hybrid composite (HC) made up of a metal oxide and 2D materials could facilitate an enhanced photocatalytic reaction and thus further extend and widen the applications of these materials [4]. Although HCs of several metal oxides and graphene/graphene oxide have been explored [4], an $\mathrm{HC}$ using gallium oxide $(\mathrm{GaO})$ and graphene oxide (GO) has not yet been investigated. In fact, $\mathrm{GaO}$ is an important metal oxide showing a larger energy bandgap $(4.8 \sim 5.1 \mathrm{eV})$ than conventional metal oxides, such as $\mathrm{TiO}_{2}(3.0 \mathrm{eV})$ and $\mathrm{ZnO}(3.2 \mathrm{eV})[11,12]$. In addition, $\mathrm{GaO}$ has recently garnered more attention for use with power electronics [13] and the extension of their applications to various other areas. In this work, a GaO-GO HC is presented, and its material and photocatalytic reaction properties are investigated. Photo-induced charged carriers are generated from the $\mathrm{GaO}$, and these carriers spread out across the $\mathrm{GO}$, which has a large surface area, resulting in a boost to the photocatalytic reaction. In addition, the $\mathrm{GO}$ that attaches to the $\mathrm{GaO}$ is able to retard the recombination of generated electron-hole pairs. 


\section{Experimental Section}

HCs made up of $\mathrm{GaO}$ and $\mathrm{GO}$ were synthesized using a conventional hydrothermal method that is often used to produce metal oxide powder [14]. $\mathrm{GaCl}_{3}(99.999 \%)$ was diluted in deionized (DI) water to form a synthesis precursor, and the GO was prepared in the DI water as a solvent. After mixing the $\mathrm{GaCl}_{3}$ and $\mathrm{GO}$ solutions, ammonium hydroxide $\left(\mathrm{NH}_{4}(\mathrm{OH}) 28 \%\right.$ in $\left.\mathrm{H}_{2} \mathrm{O}\right)$ was also added to maintain a $\mathrm{pH}$ balance above 8 since the reaction necessary to form Ga oxide nanoparticles would not occur at a $\mathrm{pH}$ value below 8 [15]. All of the processes were conducted in a three-neck round-bottom flask in an ice bath to maintain a temperature of $0{ }^{\circ} \mathrm{C}$. The solvent and $\mathrm{HC}$ were separated via centrifugal force at $4000 \mathrm{rpm}$ for $30 \mathrm{~min}$. The collected HCs were then washed and rinsed to remove the ammonia and chlorine residues. Finally, the HCs were dried in a conventional freeze-dryer at $-80^{\circ} \mathrm{C}$ for $48 \mathrm{~h}$. The formed $\mathrm{HCs}$ and $\mathrm{GaO}$ were characterized via scanning electron microscopy (SEM) (Busan, Korea), X-Ray Diffraction (XRD) (Busan, Korea) and Raman analyses (Seoul, Korea). Furthermore, the photocatalytic reactions of the HCs were evaluated via the degradation of methylene blue (MB; $\mathrm{C}_{16} \mathrm{H}_{18} \mathrm{~N}_{3} \mathrm{SCl}$ ) at $245 \mathrm{~nm}$ of exposure. Multiple 100-mg HCs were added to $100-\mathrm{mL} \mathrm{MB}$ solutions $\left(0.3 \mathrm{~g} / \mathrm{L}\right.$ in $\left.\mathrm{H}_{2} \mathrm{O}\right)$. The mixed solutions were exposed under a 245 -nm mercury lamp (Seongnam, Korea) at $0.4 \mathrm{~mW} / \mathrm{s}$ for $0 \mathrm{~min}, 60 \mathrm{~min}, 120 \mathrm{~min}$ and $180 \mathrm{~min}$, respectively. For the absorbance spectrum analysis, $5 \mathrm{~mL}$ was collected from each mixed solution, and the absorbance spectrum was obtained as a function of wavelength in the range of $450-800 \mathrm{~nm}$. The entire experiment was conducted in the dark to avoid any light source disturbance.

\section{Results and Discussion}

The HCs formed via the aforementioned processes are shown in Figure 1. The SEM images in Figure 1a,b shows grain-shaped $\mathrm{GaO}$, and Figure $1 \mathrm{~b}$ also reveals that the formed $\mathrm{GO}$ connected to the $\mathrm{GaO}$ in a fishing net configuration. The $\mathrm{GaO}$ samples with higher $\mathrm{GO}$ concentrations showed higher surface areas, which eventually led to enhancements in the photocatalytic reaction. The GaO only sample was made using a $5 \mathrm{~g} \mathrm{GaCl}_{3}$ solution. However, the $4 \% \mathrm{GO}-\mathrm{GaO}$ and $10 \% \mathrm{GO}-\mathrm{GaO}$ samples were made using $0.2 \mathrm{~g}$ of $\mathrm{GO}$ and $0.5 \mathrm{~g}$ of $\mathrm{GO}$ as well as a $5 \mathrm{~g} \mathrm{GaCl}_{3}$ solution. In addition, it is interesting to note that the height to width ratio of the $\mathrm{GaO}$ with a $\mathrm{GO} 4 \%$ solution decreased in the $\mathrm{GaO}$ with a $\mathrm{GO} 10 \%$ solution. It was presumed that the presence of $\mathrm{GO}$ might have hindered the $\mathrm{GaO}$ growth for a certain structural plane More detailed structure and crystallinity information regarding the $\mathrm{GaO}$ and GO was obtained via XRD and Raman analyses.

a
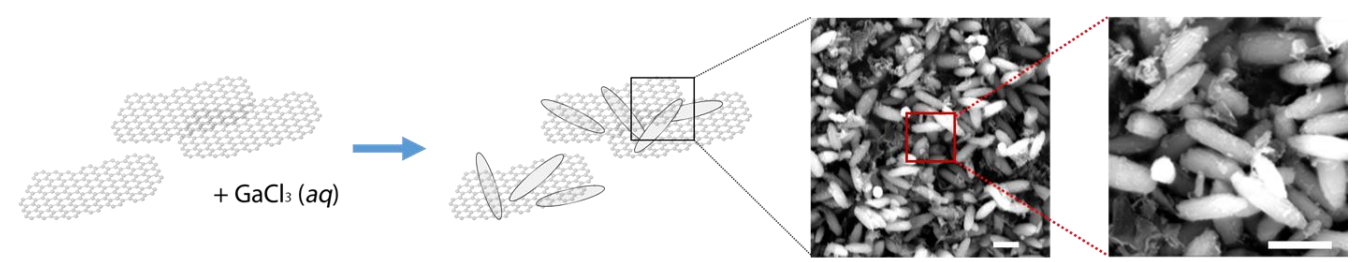

b

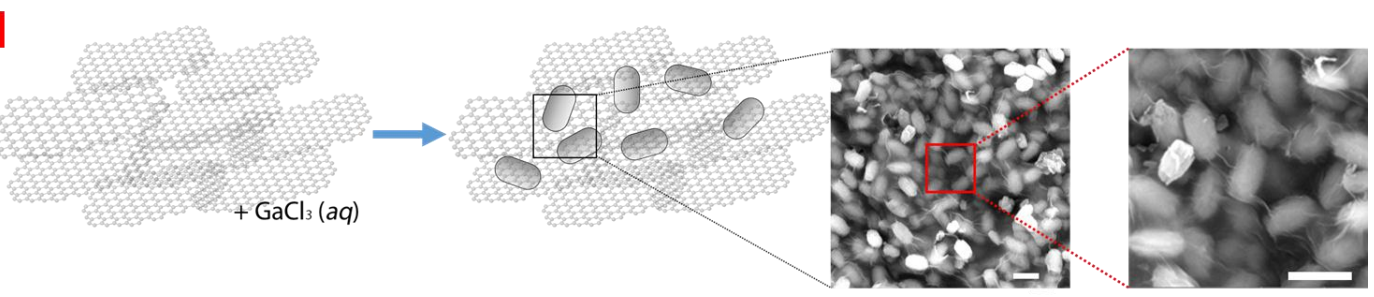

Figure 1. Schematic illustrations of hybrid composite $(\mathrm{HC})$ growth depending on (a) a $4 \%$ graphene oxide (GO) solution and (b) a 10\% GO solution. The scale bar in the scanning electron microscopy (SEM) is $1 \mu \mathrm{m}$. 
Figure 2a shows the XRD spectra of GaO-GO HCs, as well as GaO for comparison. It shows that an orthorhombic structure was preferred in the $\mathrm{GaO}$, and the crystallinity of the $\mathrm{GaO}$ was preserved even when forming HCs with GO [16].
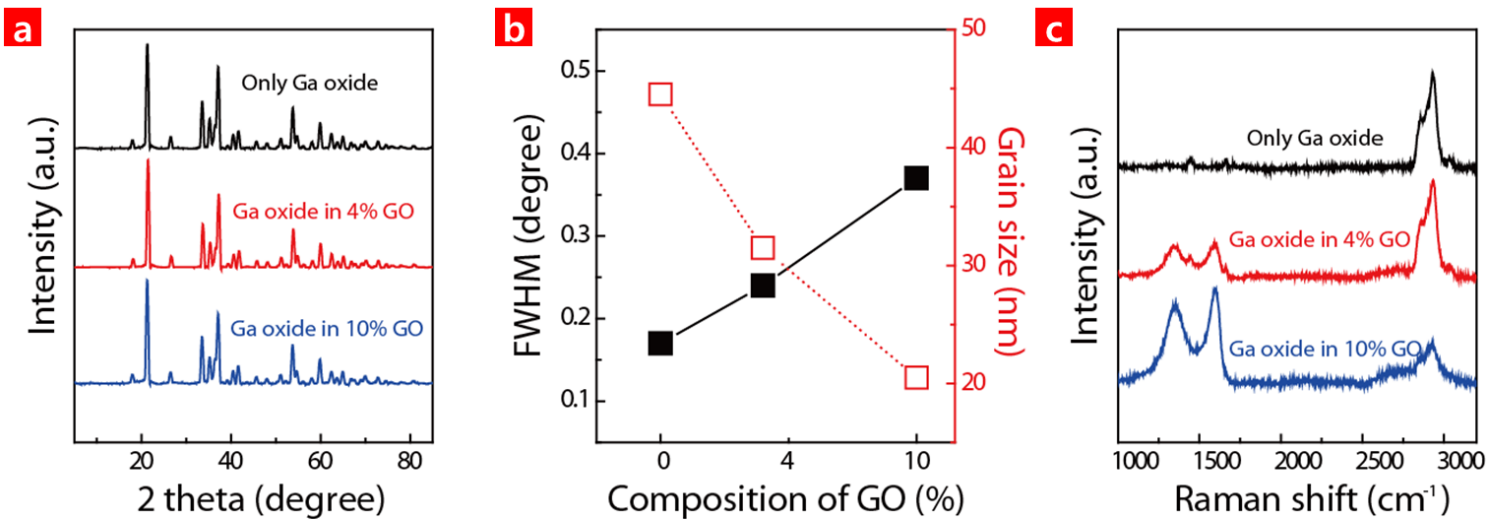

Figure 2. (a) X-Ray Diffraction (XRD) spectra of different HCs made up of gallium oxide (GaO) and with/without GO; (b) Full-width at half maximum (FWHM) variation for XRD peaks from (a) and grain size of the HCs as a function of GO concentration in the solution; (c) Raman of different HCs using the same proportions as in (a).

In addition, the full-width at half maximum (FWHM) of $\{110\}$ was measured, and the grain size of the structures was extracted from the FWHM at different GO concentrations [17]. The $\mathrm{GaO}$ grain size of $45 \mathrm{~nm}$ continued to decrease as the GO concentration increased in the HC. It was presumed that the functional groups such as hydroxyl, epoxide, carbonyl and carboxyl at the GO surface can serve as nucleation sites. Accordingly, there should be more nucleation sites for the $\mathrm{GaO}$ when there is a higher $\mathrm{GO}$ concentration because this leads to a smaller $\mathrm{GaO}$ grain size in an $\mathrm{HC}$ [18]. This reveals that the presence of GO during the Ga oxide formation affects the grain size of the Ga oxide in a nano scale range. The crystallinity of the HC was further investigated via Raman analyses, as shown in Figure 2c. The results show that the peaks representing $\mathrm{GaO}$ were observed in the $\mathrm{GaO}$ particles. As the GO concentration increased in the HC, the GO peak became more prominent. D-band (D) occurs at 1350 region because of oxide. The G-band $(G)$ is generated at 1350 region because of graphite [17]. The D/G ratio of the GO peak in the HC was comparable to that of a conventional GO. The results show that high quality GO was preserved even in the GO in the HC.

The photocatalytic reaction of the $\mathrm{GaO}$ with/without $\mathrm{GO}$ was investigated through the photo-degradation of the MB under UV exposure. Figure 3 shows time evolution of the MB absorbance spectrum depending on nanoparticle sample, i.e., only $\mathrm{GaO}, \mathrm{GaO}$ in a $4 \% \mathrm{GO}$ solution and $\mathrm{Ga}$ oxide in a 10\% GO solution. Before the spectra analysis, the samples were exposed at $254 \mathrm{~nm}$ for $0 \mathrm{~min}, 60 \mathrm{~min}$, $120 \mathrm{~min}$ and $180 \mathrm{~min}$, respectively. The results show that the absorbance spectrum of the MB with only $\mathrm{GaO}$ remained constant. However, the spectrum intensity significantly decreased according to time and GO concentration. This reveals that the MB degradation proportional to the GO concentration, i.e., the total surface area in the MB.

A schematic illustration of the charged carrier transfer and $\mathrm{MB}$ degradation mechanism of the $\mathrm{GaO}$ (Figure 4a) and the HCs (Figure 4b) made up of Ga oxide and GO is described in Figure 4. The HCs made up of $\mathrm{GaO}$ and $\mathrm{GO}$ were able to slow the recombination of generated electron-hole pairs and enhance the reaction potential with the MB. 
a

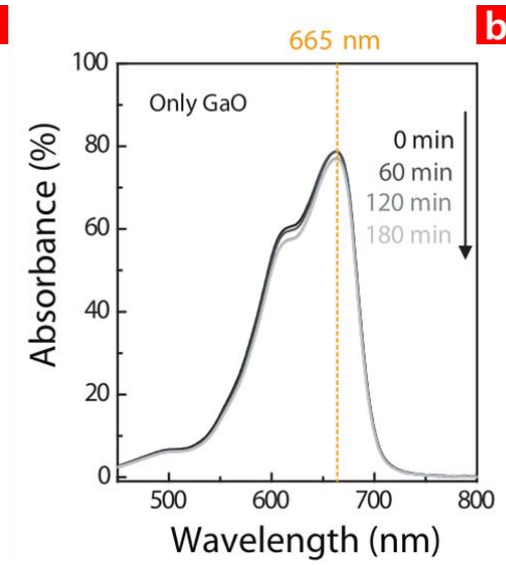

b

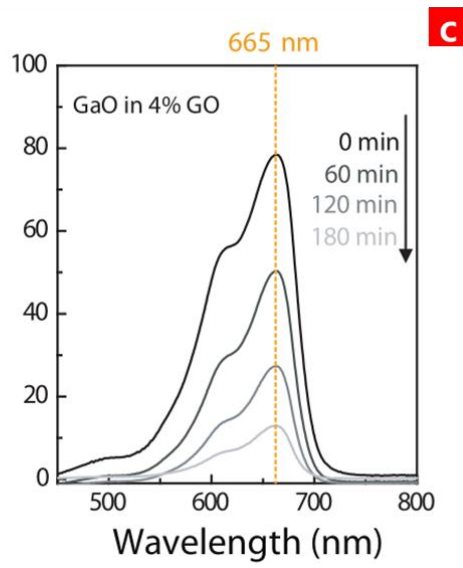

c

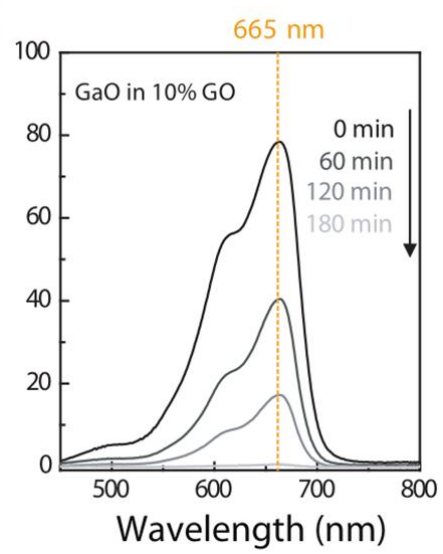

Figure 3. The absorbance spectra of the methylene blue (MB) solution where (a) $\mathrm{GaO}$; (b) an $\mathrm{HC}$ of $\mathrm{GaO}$ and $4 \% \mathrm{GO}$; and (c) an $\mathrm{HC}$ of $\mathrm{GaO}$ and $10 \% \mathrm{GO}$ were added to the MB. Each sample was exposed to $254 \mathrm{~nm}$ of radiation at different times.
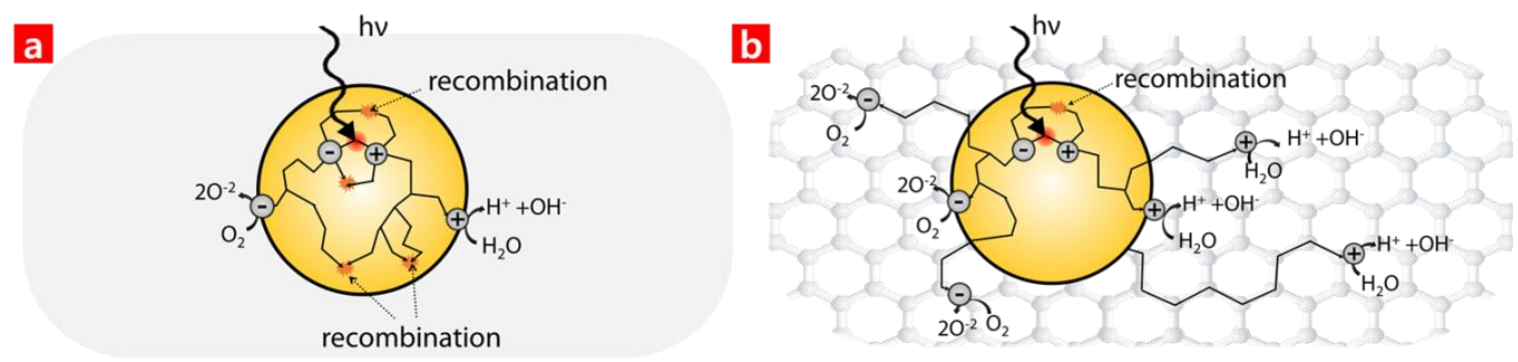

Figure 4. Schematic illustrations of charged carrier generation and transfer, as well as the water splitting mechanism of (a) the Ga oxide and (b) the HC of Ga oxide and GO. hv is ultraviolet light energy, where $\mathrm{h}$ is Planck's constant and $v$ is frequency.

\section{Conclusions}

Hybrid composites (HCs) made up of $\mathrm{GaO}$ and GO were investigated to facilitate enhanced photocatalytic reactions under ultraviolet (UV) radiation. The improvements in the reaction were attributed to the increased surface area that resulted when the $\mathrm{GO}$ attached to the $\mathrm{GaO}$. The generated charged carriers in the $\mathrm{GaO}$ under UV radiation spread out through the $\mathrm{GO}$ and retarded the recombination of electron-hole pairs, significantly enhancing the photocatalytic reaction with the MB. The material properties of both the GaO-GO were preserved even after the formation of HCs made up of $\mathrm{GaO}$ and GO. The HCs developed in this study can be used in various applications requiring enhanced photocatalytic reactions.

Acknowledgments: This work was supported by the Basic Science Research Program through the National Research Foundation of Korea (NRF) funded by the Ministry of Science, ICT \& Future Planning (2014R1A1A1004770), by the Technology Innovation Program (No. 10044410) funded by the Korea Government Ministry of Knowledge Economy, by an Energy Efficiency \& Resources of the Korea Institute of Energy Technology Evaluation and Planning (KETEP) grant funded by the Korea Government Ministry of Knowledge Economy (No. 20142010102690) and by a grant from the Advanced Technology Center R\&D Program funded by the Ministry of Trade, Industry \& Energy of Korea (10048475), by the Korea government Ministry of Knowledge Economy.

Author Contributions: S.K., K.I.H., W.S.Y. and W.S.H. conceived and designed the experiments, W.K.P. and Y.Y. contributed to ultraviolet measurements and interpretation, I.G.L. and C.S.Y. provided and interpreted the morphologic model, S.K., W.S.Y. and W.S.H. wrote the manuscript. All authors discussed the results and commented on the manuscript at all stages.

Conflicts of Interest: The authors declare no conflict of interest. 


\section{References}

1. Liu, J.; Bai, H.; Wang, Y.; Liu, Z.; Zhang, X.; Sun, D.D. Self-assembling $\mathrm{TiO}_{2}$ nanorods on large graphene oxide sheets at a two-phase interface and their anti-recombination in photocatalytic applications. Adv. Funct. Mater. 2006, 20, 4175-4181. [CrossRef]

2. Akhavan, O.; Ghaderi, E. Photocatalytic reduction of graphene oxide nanosheets on $\mathrm{TiO}_{2}$ thin film for photoinactivation of bacteria in solar light irradiation. J. Phys. Chem. C 2009, 113, 20214-20220. [CrossRef]

3. Li, D.; Zhao, Y.; Wang, Q.; Yang, Y.; Zhang, Z. Enhanced biohydrogen production by accelerating the hydrolysis of macromolecular components of waste activated sludge using $\mathrm{TiO}_{2}$ photocatalysis as a pretreatment. Open J. Appl. Sci. 2013, 3. [CrossRef]

4. Ohtani, B. Revisiting the fundamental physical chemistry in heterogeneous photocatalysis: Its thermodynamics and kinetics. Phys. Chem. Chem. Phys. 2014, 16, 1788-1797. [CrossRef] [PubMed]

5. Yoon, Y.; Park, W.K.; Hwang, T.M.; Yoon, D.H.; Yang, W.S.; Kang, J.W. Comparative evaluation of magnetite-graphene oxide and magnetite-reduced graphene oxide composite for As (III) and As (V) removal. J. Hazard. Mater. 2016, 304, 196-204. [CrossRef]

6. Kudo, A.; Mikami, I. Photocatalytic activities and photophysical properties of $\mathrm{Ga}_{2-x} \mathrm{In}_{x} \mathrm{O}_{3}$ solid solution. J. Chem. Soc. 1998, 94, 2929-2932. [CrossRef]

7. Luo, M.; Yao, W.; Huang, C.; Wu, Q.; Xu, Q. Shape effects of Pt nanoparticles on hydrogen production via $\mathrm{Pt} / \mathrm{CdS}$ photocatalysts under visible light. J. Mater. Chem. A 2015, 3, 13884-13891. [CrossRef]

8. Ben-Shahar, Y.; Scotognella, F.; Kriegel, I.; Moretti, L.; Cerullo, G.; Rabani, E.; Banin, U. Optimal metal domain size for photocatalysis with hybrid semiconductor-metal nanorods. Nat. Commun. 2016, 7. [CrossRef]

9. Girija, K.; Thirumalairajan, S.; Patra, A.K.; Mangalaraj, D.; Ponpandian, N.; Viswanathan, C. Organic additives assisted synthesis of mesoporous $\beta-\mathrm{Ga}_{2} \mathrm{O}_{3}$ nanostructures for photocatalytic dye degradation. Semicond. Sci. Technol. 2013, 28. [CrossRef]

10. Park, S.; Ruoff, R.S. Chemical methods for the production of graphenes. Nat. Nanotechnol. 2009, 4, $217-224$. [CrossRef] [PubMed]

11. Scaife, D.E. Oxide semiconductors in photoelectrochemical conversion of solar energy. Sol. Energy 1980, 25, 41-54. [CrossRef]

12. Zhang, H.; Ji, Z.; Xia, T.; Meng, H.; Low-Kam, C.; Liu, R.; Wang, M.; Lin, S.; Wang, X.; Liao, Y.-P.; et al. Use of metal oxide nanoparticle band gap to develop a predictive paradigm for oxidative stress and acute pulmonary inflammation. ACS Nano 2012, 6, 4349-4368. [CrossRef] [PubMed]

13. Hwang, W.S.; Verma, A.; Peelaers, H.; Protasenko, V.; Rouvimov, S.; Xing, H.G.; Albrecht, M.; Seabaugh, A.; Haensch, W.; van de Walle, C.; et al. High-voltage field effect transistors with wide-bandgap $\beta-\mathrm{Ga}_{2} \mathrm{O}_{3}$ nanomembranes. Appl. Phys. Lett. 2014, 104. [CrossRef]

14. Zhang, J.; Liu, Z.; Lin, C.; Lin, J. A simple method to synthesize $\beta-\mathrm{Ga}_{2} \mathrm{O}_{3}$ nanorods and their photoluminescence properties. J. Cryst. Growth 2005, 280, 99-106. [CrossRef]

15. Ghazali, N.M.; Mahmood, M.R.; Yasui, K.; Hashim, A.M. Electrochemically deposited gallium oxide nanostructures on silicon substrates. Nanoscale Res. Lett. 2014, 9. [CrossRef] [PubMed]

16. Wang, H.; Robinson, J.T.; Diankov, G.; Dai, H. Nanocrystal growth on graphene with various degrees of oxidation. J. Am. Chem. Soc. 2010, 132, 3270-3271. [CrossRef]

17. Kumar, Y.A.; Chandkiram, G.; Prabhakar, S. Crystallization kinematics and dielectric behavior of (Ba, Sr) $\mathrm{TiO}_{3}$ borosilicate glass ceramics. New J. Glass Cerami. 2012, 2. [CrossRef]

18. Dreyer, D.R.; Park, S.; Bielawski, C.W.; Ruoff, R.S. The chemistry of graphene oxide. Chem. Soc. Rev. 2010, 39, 228-240. [CrossRef]

(C) 2016 by the authors; licensee MDPI, Basel, Switzerland. This article is an open access article distributed under the terms and conditions of the Creative Commons Attribution (CC-BY) license (http:/ / creativecommons.org/licenses/by/4.0/). 